Article

\title{
Fuzzy Differential Subordinations Obtained Using a Hypergeometric Integral Operator
}

\author{
Georgia Irina Oros
}

check for

updates

Citation: Oros, G.I. Fuzzy

Differential Subordinations Obtained Using a Hypergeometric Integral Operator. Mathematics 2021, 9, 2539.

https://doi.org/10.3390/math9202539

Academic Editors: Ioan Dzitac and Sorin Nadaban

Received: 14 September 2021

Accepted: 6 October 2021

Published: 10 October 2021

Publisher's Note: MDPI stays neutral with regard to jurisdictional claims in published maps and institutional affiliations.

Copyright: (C) 2021 by the author. Licensee MDPI, Basel, Switzerland. This article is an open access article distributed under the terms and conditions of the Creative Commons Attribution (CC BY) license (https:// creativecommons.org/licenses/by/ $4.0 /)$.
Department of Mathematics and Computer Science, University of Oradea, 1 Universitatii Street, 410087 Oradea, Romania; georgia_oros_ro@yahoo.co.uk or goros@uoradea.ro

\begin{abstract}
This paper is related to notions adapted from fuzzy set theory to the field of complex analysis, namely fuzzy differential subordinations. Using the ideas specific to geometric function theory from the field of complex analysis, fuzzy differential subordination results are obtained using a new integral operator introduced in this paper using the well-known confluent hypergeometric function, also known as the Kummer hypergeometric function. The new hypergeometric integral operator is defined by choosing particular parameters, having as inspiration the operator studied by Miller, Mocanu and Reade in 1978. Theorems are stated and proved, which give corollary conditions such that the newly-defined integral operator is starlike, convex and close-to-convex, respectively. The example given at the end of the paper proves the applicability of the obtained results.
\end{abstract}

Keywords: analytic function; univalent function; fuzzy differential subordination; fuzzy best dominant; confluent hypergeometric function; integral operator

\section{Introduction}

The introduction of the fuzzy set concept by Lotfi A. Zadeh, in the paper "Fuzzy Sets" [1] in 1965, did not suggest the extraordinary evolution of the concept which followed. Received with distrust at first, the concept is very popular nowadays, being adapted to many research topics. Mathematicians were also interested in embedding the concept of fuzzy set in their research and it was indeed included in many mathematical approaches. The review paper included in the present special issue, devoted to the celebration of the 100th anniversary of Zadeh's birth [2], shows how fuzzy set theory has evolved related to certain branches of science, and points out the contribution of one of Zadeh's disciples, Professor I. Dzitac, to the development of soft computing methods connected with fuzzy set theory. Professor I. Dzitac has celebrated his friendship with the multidisciplinary scientist, Lotfi A. Zadeh, by writing the introductory paper of a special issue on fuzzy logic dedicated to the centenary of Zadeh's birth [3].

As far as complex analysis is concerned, fuzzy set theory has been included in studies related to geometric function theory in 2011, when the first paper appeared introducing the notion of subordination in fuzzy set theory [4] which has had its inspiration in the classical aspects of subordination introduced by Miller and Mocanu [5,6]. The next papers published followed the line of research set by Miller and Mocanu and referred to fuzzy differential subordination, adapting notions from the already well-established theory of differential subordination [7-9]. The idea was soon picked up by researchers in geometric function theory and all the classical lines of research in this topic were adapted to the new fuzzy aspects. A review paper published in 2017 [10] included in its references the first published papers related to this topic, validating its development. The dual notion of fuzzy differential superordination was also introduced in 2017 [11].

An important topic in geometric function theory is conducting studies which involve operators. Such studies for obtaining new fuzzy subordination results were published soon after the notion was introduced, in 2013 [12], continued during the next years [13-16] and 
later added the superordination results [17-19]. During the last years, many papers were published which show that the research on this topic is in continuous development process and we mention only a few here [20-24].

Following this line of research, a new hypergeometric integral operator is introduced in this paper using a confluent (or Kummer) hypergeometric function and having, as inspiration, the operator studied by Miller, Mocanu and Reade in 1978, by taking specific values for parameters involved in its definition. Fuzzy differential subordinations are obtained and the fuzzy best dominants are given, which facilitate obtaining sufficient conditions for univalence of this operator.

\section{Preliminaries}

The research presented in this paper is done in the general environment known in the theory of differential subordination given in the monograph [25] combined with fuzzy set notions introduced in $[4,7]$.

The unit disc of the complex plane is denoted by $U . \mathcal{H}(U)$ stands for the class of holomorphic functions in $U$. Consider the subclass, $\mathcal{A}_{n}=\{f \in \mathcal{H}(U): f(z)=$ $\left.z+a_{n+1} z^{n+1}+\cdots, z \in U\right\}$, with $\mathcal{A}_{1}=\mathcal{A}$.

For $a \in \mathbb{C}, n \in \mathbb{N}^{*}$ the following subclass of holomorphic functions is obtained: $\mathcal{H}[a, n]=\left\{f \in \mathcal{H}(U): f(z)=a+a_{n} z^{n}+a_{n+1} z^{n+1}+\cdots, z \in U\right\}$, with $\mathcal{H}_{0}=\mathcal{H}[0,1]$.

For $\alpha<1$, let $\mathcal{S}^{*}(\alpha)=\left\{f \in \mathcal{A}: \operatorname{Re}\left(\frac{z f^{\prime}(z)}{f(z)}\right)>\alpha\right\}$ denote the class of starlike functions of order $\alpha$. For $\alpha=0$, the class of starlike functions is denoted by $\mathcal{S}^{*}$.

For $\alpha<1$, let $\mathcal{K}(\alpha)=\left\{f \in \mathcal{A}: \operatorname{Re}\left(\frac{z f^{\prime \prime}(z)}{f^{\prime}(z)}+1\right)>\alpha\right\}$ denote the class of convex functions of order $\alpha$. For $\alpha=0$, the class of convex functions is denoted by $\mathcal{K}$.

The subclass of close-to-convex functions is defined as: $\mathcal{C}=\{f \in \mathcal{H}(U): \exists \varphi \in \mathcal{K}$, $\left.\operatorname{Re}\left(\frac{f^{\prime}(z)}{\varphi^{\prime}(z)}\right)>0, z \in U\right\}$.

It is also said that function $f$ is close-to-convex with respect to function $\varphi$.

Definition 1 ([4]). Let $D \subset \mathbb{C}$ and $z_{0} \in D$ be a fixed point. We take the functions $f, g \in \mathcal{H}(D)$. The function $f$ is said to be fuzzy subordinate to $g$ and write $f \prec \mathcal{F} g$ or $f(z) \prec \mathcal{F} g(z)$, if there exists a function $F: \mathbb{C} \rightarrow[0,1]$, such that

(i) $f\left(z_{0}\right)=g\left(z_{0}\right)$,

(ii) $F(f(z)) \leq F(g(z))$, for all $z \in D$.

Remark 1. (a) Such a function $F: \mathbb{C} \rightarrow[0,1]$ can be considered $F(z)=\frac{|z|}{1+|z|}, F(z)=\frac{1}{1+|z|}$, $F(z)=\frac{\sqrt{|z|}}{1+\sqrt{|z|}}$.

(b) Relation (ii) is equivalent to $f(D) \subset g(D)$.

Definition 2 ([7], Definition 2.2). Let $\psi: \mathbb{C}^{3} \times D \rightarrow \mathbb{C}, a \in \mathbb{C}$, and let $h$ be univalent in $U$, with $h\left(z_{0}\right)=a, g$ be univalent in $D$, with $g\left(z_{0}\right)=a$, and $p$ be analytic in $D$, with $p\left(z_{0}\right)=a$. Likewise, $\psi\left(p(z), z p^{\prime}(z), z^{2} p^{\prime \prime}(z) ; z\right)$ is analytic in $D$ and $F: \mathbb{C} \rightarrow[0,1], F(z)=\frac{|z|}{1+|z|}$. If $p$ is analytic in $D$ and satisfies the (second-order) fuzzy differential subordination

$$
F\left(\psi\left(p(z), z p^{\prime}(z), z^{2} p^{\prime \prime}(z) ; z\right)\right) \leq F(h(z)), \quad z \in U
$$

i.e., $\psi\left(p(z), z p^{\prime}(z), z^{2} p^{\prime \prime}(z) ; z\right) \prec \mathcal{F} h(z)$, or

$$
\frac{\left|\psi\left(p(z), z p^{\prime}(z), z^{2} p^{\prime \prime}(z) ; z\right)\right|}{1+\left|\psi\left(p(z), z p^{\prime}(z), z^{2} p^{\prime \prime}(z) ; z\right)\right|} \leq \frac{|h(z)|}{1+|h(z)|}, z \in D,
$$

then $p$ is called a fuzzy solution of the fuzzy differential subordination. The univalent function $q$ is called a fuzzy dominant of fuzzy solutions of the differential subordination, or more simply, a fuzzy 
dominant, if $\frac{|p(z)|}{1+|p(z)|} \leq \frac{|q(z)|}{1+|q(z)|}$, or $p(z) \prec_{\mathcal{F}} q(z), z \in D$, for all $p$ satisfying (1) or (2). A fuzzy dominant $\widetilde{q}$ that satisfies $\frac{|\widetilde{q}(z)|}{1+|\widetilde{q}(z)|} \leq \frac{|q(z)|}{1+|q(z)|}$, or $\widetilde{q}(z) \prec_{\mathcal{F}} q(z), z \in D$, for all fuzzy dominants $q$ of (1) or (2) is said to be the fuzzy best dominant of (1) or (2). Note that the fuzzy best dominant is unique up to a rotation in $D$.

Lemma 1 ([25], Theorem 2.2). Let $\delta, \omega \in \mathbb{C}, \omega \neq 0$, and $h$ be a convex function in $D$, and $F$ : $\mathbb{C} \rightarrow[0,1], F(z)=\frac{|z|}{1+|z|}, z \in D$. We suppose that the Briot-Bouquet differential equation

$$
q(z)+\frac{z q^{\prime}(z)}{\delta+\omega q(z)}=h(z), z \in D, q\left(z_{0}\right)=h\left(z_{0}\right)=a
$$

has a solution $q \in \mathcal{H}(D)$, which verifies $q(z) \prec_{\mathcal{F}} h(z), z \in D$, or $\frac{|q(z)|}{1+|q(z)|} \leq \frac{|h(z)|}{1+\mid h(z)}, z \in D$.

If the function $p \in \mathcal{H}[a, n], \psi: \mathbb{C}^{2} \times D \rightarrow \mathbb{C}, \psi\left(p(z), z p^{\prime}(z)\right)=p(z)+\frac{z p^{\prime}(z)}{\delta+\omega p(z)}$ is analytic in $D$, with $\psi\left(p\left(z_{0}\right), z_{0} p^{\prime}\left(z_{0}\right)\right)=h\left(z_{0}\right), z_{0} \in D$, then

$$
\psi\left(p(z), z p^{\prime}(z)\right) \prec_{\mathcal{F}} h(z), z \in D,
$$

or

$$
\frac{\left|\psi\left(p(z), z p^{\prime}(z)\right)\right|}{1+\left|\psi\left(p(z), z p^{\prime}(z)\right)\right|} \leq \frac{|h(z)|}{1+|h(z)|}
$$

implies

$$
p(z) \prec_{\mathcal{F}} q(z) \text {, or } \frac{|p(z)|}{1+|p(z)|} \leq \frac{|q(z)|}{1+|q(z)|}, z \in D,
$$

and $q$ is the fuzzy best dominant of the fuzzy differential subordination (3) or (4).

The confluent (or Kummer) hypergeometric function has been investigated connected to univalent functions more intensely starting from 1985 when it was used by L. de Branges in the proof of Bieberbach's conjecture [26]. The applications of hypergeometric functions in univalent function theory is very well pointed out in the review paper, recently published by H.M. Srivastava [27].

Definition 3 ([25]). Let $u$ and $v$ be complex numbers with $v \neq 0,-1,-2, \ldots$, and consider the function defined by

$$
\begin{gathered}
\phi(u, v ; z)=\frac{\Gamma(v)}{\Gamma(u)} \sum_{k=0}^{\infty} \frac{\Gamma(u+k)}{\Gamma(v+k)} \frac{z^{k}}{k !}= \\
1+\frac{u}{v} \frac{z}{1 !}+\frac{u(u+1)}{v(v+1)} \frac{z^{2}}{2 !}+\cdots+\frac{u(u+1) \ldots(u+n-1)}{v(v+1) \ldots(v+n-1)} \frac{z^{n}}{n !}+\ldots
\end{gathered}
$$

where $(e)_{k}=\frac{\Gamma(e+k)}{\Gamma(e)}=e(e+1)(e+2) \ldots(e+k-1)$, and $(e)_{0}=1$, called the confluent (or Kummer) hypergeometric function is analytic in $\mathbb{C}$.

Remark 2. (a) For $z=0, \phi(u, v ; 0)=1$ and $\phi(u, v ; z) \neq 0, z \in U$,

(b) For $u \neq 0, \phi^{\prime}(u, v ; 0)=\frac{u}{v} \neq 0$.

The operator used for obtaining the original results presented in this paper was obtained using a confluent (or Kummer) hypergeometric function and a general operator studied in 1978 by S.S. Miller, P.T. Mocanu and M.O. Reade [28] by taking specific values for parameters $\beta, \gamma, \alpha, \delta$ :

$$
J(f)(z)=\left[\frac{\beta+\gamma}{z^{\gamma} \phi(z)} \int_{0}^{z} f^{\alpha}(t) \varphi(t) t^{\delta-1} d t\right]^{\frac{1}{\beta}} .
$$


A confluent (or Kummer) hypergeometric function was recently used in many papers for defining new interesting operators as it can be seen in [29-32].

Two more lemmas from differential subordination theory that are necessary in the proofs of the original results are listed next:

Lemma 2 ([33], Theorem 4.6.3, p. 84). A necessary and sufficient condition for a function $f \in \mathcal{H}(U)$ to be close-to-convex is given by:

$$
\int_{\theta_{1}}^{\theta_{2}} \operatorname{Re}\left[1+\frac{z f^{\prime \prime}(z)}{f^{\prime}(z)}\right] d \theta>-\pi, z_{0}=r_{0} e^{i \theta_{0}},
$$

for all $\theta_{1}, \theta_{2}$ with $0 \leq \theta_{1}<\theta_{2}<2 \pi, r \in(0,1)$.

Lemma 3 ([25], Theorem Marx-Strohhäcker, p. 9). If $f \in \mathcal{K}$ then $\operatorname{Re} \frac{z f^{\prime}(z)}{f(z)}>\frac{1}{2}$, i.e., $f \in$ $\mathcal{S}^{*}\left(\frac{1}{2}\right)$, for $z \in U$.

\section{Main Results}

The new hypergeoemtric integral operator is defined using Definition 3 and the integral operator given by relation (6).

Definition 4. Let $\beta>1, \gamma>0$ and the confluent (Kummer) hypergeometric function $\phi$ given by (5).

Let $M: \mathcal{H}(U) \rightarrow \mathcal{H}(U)$ be given by:

$$
M(z)=\frac{\beta}{z^{\beta-1}} \int_{0}^{z}\left[\frac{\Gamma(v)}{\Gamma(u)} \sum_{k=0}^{\infty} \frac{\Gamma(u+k)}{\Gamma(v+k)} \frac{t^{k}}{k !}\right] t^{\beta-1} d t, \quad z \in U .
$$

Remark 3. (a) For $\beta>1, \gamma>0$ and $\phi(u, v ; z)=\frac{\Gamma(v)}{\Gamma(u)} \sum_{k=0}^{\infty} \frac{\Gamma(u+k)}{\Gamma(v+k)} \frac{z^{k}}{k !}=1+\frac{u}{v} \frac{z}{1 !}+\frac{u(u+1)}{v(v+1)} \frac{z^{2}}{2 !}+$ $\ldots, u, v \in \mathbb{C}, v \neq 0,-1,-2, \ldots$, we have

$$
\begin{gathered}
M(z)=\frac{\beta}{z^{\beta-1}} \int_{0}^{z}\left[1+\frac{u}{v} \frac{t}{1 !}+\frac{u(u+1)}{v(v+1)} \frac{t^{2}}{2 !}+\ldots\right]^{\gamma} t^{\beta-1} d t= \\
\frac{\beta}{z^{\beta-1}} \int_{0}^{z}\left(1+\gamma \frac{u}{v} t+p_{2} t^{2}+\ldots\right) t^{\beta-1} d t= \\
\frac{\beta}{z^{\beta-1}} \int_{0}^{z}\left[t^{\beta-1}+\gamma \frac{u}{v} t^{\beta}+\ldots\right] d t=z+\gamma \frac{u}{v} \frac{\beta}{\beta+1} z^{2}+p_{2} \frac{\beta}{\beta+2} z^{3}+\ldots,
\end{gathered}
$$

which is the analytic expression of the operator $M$.

(b) For $z \in U, M^{\prime}(z)=1+2 \gamma \frac{u}{v} \frac{\beta}{\beta+1} z+\ldots$, with $M^{\prime}(0)=1$.

Using the method of differential subordination, next, a theorem is proved, giving the best dominant of a certain fuzzy differential subordination. Using specific functions as the fuzzy best dominant, conditions for starlikeness and convexity of the operator $M$ are obtained as corollaries.

Theorem 1. For $\beta, \gamma \in \mathbb{C}, \beta>1, \gamma>0$, let the fuzzy function $F: \mathbb{C} \rightarrow[0,1]$ be given by

$$
F(z)=\frac{|z|}{1+|z|}, z \in U,
$$


and consider a holomorphic function in $U$ given by the equation

$$
h(z)=q(z)+\frac{z q^{\prime}(z)}{\beta-1+\gamma q(z)}, h(0)=q(0),
$$

when $q$ is a univalent solution in $U$ which satisfies the fuzzy differential subordination:

$$
\frac{|q(z)|}{1+|q(z)|} \leq \frac{|h(z)|}{1+|h(z)|} \text { i.e., } q(z) \prec_{\mathcal{F}} h(z), z \in U \text {. }
$$

Consider $\phi(u, v ; z)$, a confluent (or Kummer) hypergeometric function given by (5) and the operator $M(z)$ given by (7).

If $1+\gamma \frac{z \phi^{\prime}(u, v ; z)}{\phi(u, v ; z)}$ is analytic in $U$, and

$$
\frac{\left|1+\gamma \frac{z \phi^{\prime}(u, v ; z)}{\phi(u, v ; z)}\right|}{1+\left|1+\gamma \frac{z \phi^{\prime}(u, v ; z)}{\phi(u, v ; z)}\right|} \leq \frac{|h(z)|}{1+|h(z)|} \text { i.e., } 1+\gamma \frac{z \phi^{\prime}(u, v ; z)}{\phi(u, v ; z)} \prec_{\mathcal{F}} h(z),
$$

then

$$
\frac{\left|\frac{z M^{\prime}(z)}{M(z)}\right|}{1+\left|\frac{z M^{\prime}(z)}{M(z)}\right|} \leq \frac{|q(z)|}{1+|q(z)|} \text { i.e., } \frac{z M^{\prime}(z)}{M(z)} \prec_{\mathcal{F}} q(z), z \in U,
$$

and $q$ is the fuzzy best dominant.

Proof. Relation (7) is equivalent to

$$
z^{\beta-1} M(z)=\beta \int_{0}^{z} \phi^{\gamma}(u, v ; t) t^{\beta-1} d t .
$$

Differentiating (13) and after short calculation we obtain

$$
(\beta-1) M(z)+z M^{\prime}(z)=\beta \phi^{\gamma}(u, v ; z) \cdot z,
$$

which is equivalent to

$$
M(z)\left[\beta-1+\frac{z M^{\prime}(z)}{M(z)}\right]=\beta \phi^{\gamma}(u, v ; z) \cdot z .
$$

We let

$$
p(z)=\frac{z M^{\prime}(z)}{M(z)}=\frac{z\left(1+2 \gamma \frac{u}{v} \frac{\beta}{\beta+1} z+\ldots\right)}{z\left(1+\gamma \frac{u}{v} \frac{\beta}{\beta+1} z+\ldots\right)}=\frac{1+2 \gamma \frac{u}{v} \frac{\beta}{\beta+1} z+\ldots}{1+\gamma \frac{u}{v} \frac{\beta}{\beta+1} z+\ldots}
$$

$p(0)=1$.

Using (16) in (15), we get

$$
M(z)[\beta-1+p(z)]=\beta \phi^{\gamma}(u, v ; z) \cdot z .
$$

Differentiating (17), we obtain

$$
\frac{z M^{\prime}(z)}{M(z)}+\frac{z p^{\prime}(z)}{\beta-1+p(z)}=\gamma \frac{z \phi^{\prime}(u, v ; z)}{\phi(u, v ; z)}+1 .
$$

Using (16) in (18), we have

$$
p(z)+\frac{z p^{\prime}(z)}{\beta-1+p(z)}=\gamma \frac{z \phi^{\prime}(u, v ; z)}{\phi(u, v ; z)}+1 .
$$


Using (19) in (12), we get

$$
\frac{\left|p(z)+\frac{z p^{\prime}(z)}{\beta-1+p(z)}\right|}{1+\left|p(z)+\frac{z p^{\prime}(z)}{\beta-1+p(z)}\right|} \leq \frac{|h(z)|}{1+|h(z)|}, z \in U
$$

In order to obtain the desired relation, Lemma 1 will be applied. To apply the lemma, let the function $\psi: \mathbb{C}^{2} \times U \rightarrow \mathbb{C}$,

$$
\psi(r, s ; z)=r+\frac{s}{\beta-1+r}, r, s \in \mathbb{C} .
$$

For $r=p(z), s=z p^{\prime}(z)$, relation (21) becomes

$$
\psi\left(p(z), z p^{\prime}(z)\right)=p(z)+\frac{z p^{\prime}(z)}{\beta-1+p(z)}, \quad z \in U .
$$

Using (22) in (20), we have

$$
\frac{\left|\psi\left(p(z), z p^{\prime}(z)\right)\right|}{1+\left|\psi\left(p(z), z p^{\prime}(z)\right)\right|} \leq \frac{|h(z)|}{1+|h(z)|}, z \in U .
$$

Applying Lemma 1, for $\delta=\beta-1, \omega=\gamma \neq 0$, we obtain

$$
\psi\left(p(z), z p^{\prime}(z)\right) \prec_{\mathcal{F}} h(z), z \in U .
$$

Using (22) in (24), we get

$$
p(z)+\frac{z p^{\prime}(z)}{\beta-1+p(z)} \prec_{\mathcal{F}} h(z), z \in U .
$$

According to Lemma 1, relation (25) implies

$$
p(z) \prec_{\mathcal{F}} q(z), z \in U .
$$

Using (16) in (26) we have

$$
\frac{z M^{\prime}(z)}{M(z)} \prec \mathcal{F} q(z), z \in U .
$$

Since $q$ satisfies the differential Equation (10), $q$ is the fuzzy best dominant.

Remark 4. Using particular expressions for the fuzzy best dominant $q$, sufficient conditions for starlikeness of the operator $M(z)$ given by (7) can be obtained.

If in Theorem 1, function $q(z)=\frac{1-z}{1+z}$ is considered the following corollary is obtained.

Corollary 1. For $\beta, \gamma \in \mathbb{C}, \beta>1, \gamma>0$, let the fuzzy function $F: \mathbb{C} \rightarrow[0,1]$ given by (9) and consider a holomorphic function in $U$ given by the equation

$$
h(z)=\frac{(\beta-1)\left(1-z^{2}\right)+1-4 z+z^{2}}{(\beta-1)(1+z)^{2}+1-z^{2}},
$$

$h(0)=q(0)=1$, where function $q(z)=\frac{1-z}{1+z}$ is a univalent solution in $U$, which satisfies the fuzzy differential subordination

$$
\frac{|1-z|}{|1+z|+|1-z|} \leq \frac{\left|(\beta-1)\left(1-z^{2}\right)+1-4 z+z^{2}\right|}{\left|(\beta-1)(1+z)^{2}+1-z^{2}\right|+\left|(\beta-1)\left(1-z^{2}\right)+1-4 z+z^{2}\right|}
$$


i.e.,

$$
\frac{1-z}{1+z} \prec_{\mathcal{F}} \frac{(\beta-1)\left(1-z^{2}\right)+1-4 z+z^{2}}{(\beta-1)(1+z)^{2}+1-z^{2}}, z \in U .
$$

Consider $\phi(u, v ; z)$ to be a confluent (or Kummer) hypergeometric operator given by (5), and the operator $M(z)$ given by (7).

$$
\begin{aligned}
& \text { If } 1+\gamma \frac{z \phi^{\prime}(u, v ; z)}{\phi(u, v ; z)} \text { is analytic in } U \text {, and } \\
& \frac{\left|1+\gamma \frac{z \phi^{\prime}(u, v ; z)}{\phi(u, v ; z)}\right|}{1+\left|1+\gamma \frac{z \phi^{\prime}(u, v ; z)}{\phi(u, v ; z)}\right|} \leq \frac{\left|(\beta-1)\left(1-z^{2}\right)+1-4 z+z^{2}\right|}{\left|(\beta-1)(1+z)^{2}+1-z^{2}\right|+\left|(\beta-1)\left(1-z^{2}\right)+1-4 z+z^{2}\right|} \\
& \text { i.e., } 1+\gamma \frac{z \phi^{\prime}(u, v ; z)}{\phi(u, v ; z)} \prec_{\mathcal{F}} \frac{(\beta-1)\left(1-z^{2}\right)+1-4 z+z^{2}}{(\beta-1)(1+z)^{2}+1-z^{2}},
\end{aligned}
$$

then

$$
\frac{\left|\frac{z M^{\prime}(z)}{M(z)}\right|}{1+\left|\frac{z M^{\prime}(z)}{M(z)}\right|} \leq \frac{\left|\frac{1-z}{1+z}\right|}{1+\left|\frac{1-z}{1+z}\right|} \text { i.e., } \frac{z M^{\prime}(z)}{M(z)} \prec_{\mathcal{F}} \frac{1-z}{1+z}, z \in U \text {, or } M \in \mathcal{S}^{*}
$$

and $q(z)=\frac{1-z}{1+z}$ is the fuzzy best dominant.

Proof. By using the function $q(z)=\frac{1-z}{1+z}$ in relation (27) from the proof of Theorem 1, the following fuzzy subordination is obtained:

$$
\frac{z M^{\prime}(z)}{M(z)} \prec_{\mathcal{F}} q(z)=\frac{1-z}{1+z}, z \in U
$$

Since $\operatorname{Re}\left(\frac{z q^{\prime \prime}(z)}{q^{\prime}(z)}+1\right)=\frac{1-\rho^{2}}{1+2 \rho \cos \alpha+\rho^{2}}>0,0<\rho<1$, the $q$ is convex, and $\operatorname{Re} \frac{1-z}{1+z}>0$, $z \in U$, differential subordination (28) is equivalent to

$$
\operatorname{Re} \frac{z M^{\prime}(z)}{M(z)}>\operatorname{Re} \frac{1-z}{1+z}>0, z \in U, \text { hence } M \in \mathcal{S}^{*} .
$$

Remark 5. Using the convex function $q(z)=\frac{1+z}{1-z}$ as the fuzzy best dominant in Theorem 1, sufficient conditions for the convexity of the operator $M(z)$ given by (7) can be obtained as a corollary.

Corollary 2. For $\beta, \gamma \in \mathbb{C}, \beta>1, \gamma>0$, let the fuzzy function $F: \mathbb{C} \rightarrow[0,1]$ given by (9) and consider a holomorphic function in $U$ given by the equation

$$
h(z)=\frac{(\beta-1)\left(1-z^{2}\right)+\gamma(1+z)^{2}}{(\beta-1)(1-z)^{2}+\gamma\left(1-z^{2}\right)}
$$

$h(0)=q(0)=1$, where function $q(z)=\frac{1+z}{1-z}$ is a univalent solution in $U$ which satisfies the fuzzy differential subordination

$$
\frac{|1+z|}{|1-z|+|1+z|} \leq \frac{\left|(\beta-1)\left(1-z^{2}\right)+\gamma(1+z)^{2}\right|}{\left|(\beta-1)(1-z)^{2}+\gamma\left(1-z^{2}\right)\right|+\left|(\beta-1)\left(1-z^{2}\right)+\gamma(1+z)^{2}\right|}
$$

i.e.,

$$
\frac{1+z}{1-z} \prec_{\mathcal{F}} \frac{(\beta-1)\left(1-z^{2}\right)+\gamma(1+z)^{2}}{(\beta-1)(1-z)^{2}+\gamma\left(1-z^{2}\right)}, \quad z \in U .
$$


Consider $\phi(u, v ; z)$ the confluent (or Kummer) hypergeometric operator given by (5), and the operator $M(z)$ given by (7).

If

$$
1+(\gamma-1) \frac{z \phi^{\prime}(u, v ; z)}{\phi(u, v ; z)}+\frac{(\gamma+1) z \phi^{\prime}(u, v ; z)+z^{2} \gamma \phi^{\prime \prime}(u, v ; z)}{\phi(u, v ; z)+\gamma z \phi^{\prime}(u, v ; z)}=E(u, v ; z)
$$

is analytic in $U$, and

$$
\begin{aligned}
\frac{|E(u, v ; z)|}{1+|E(u, v ; z)|} \leq & \frac{\left|(\beta-1)\left(1-z^{2}\right)+\gamma(1+z)^{2}\right|}{\left|(\beta-1)(1-z)^{2}+\gamma\left(1-z^{2}\right)\right|+\left|(\beta-1)\left(1-z^{2}\right)+\gamma(1+z)^{2}\right|} \\
& \text { i.e., } E(u, v ; z) \prec \mathcal{F} \frac{(\beta-1)\left(1-z^{2}\right)+\gamma(1+z)^{2}}{(\beta-1)(1-z)^{2}+\gamma\left(1-z^{2}\right)},
\end{aligned}
$$

then

$$
\frac{\left|1+\frac{z M^{\prime \prime}(z)}{M^{\prime}(z)}\right|}{1+\left|1+\frac{z M^{\prime \prime}(z)}{M^{\prime}(z)}\right|} \leq \frac{\left|\frac{1+z}{1-z}\right|}{1+\left|\frac{1+z}{1-z}\right|} \text { i.e., } 1+\frac{z M^{\prime \prime}(z)}{M^{\prime}(z)} \prec_{\mathcal{F}} \frac{1+z}{1-z}, z \in U \text {, or } M \in \mathcal{K}
$$

and $q(z)=\frac{1+z}{1-z}$ is the fuzzy best dominant.

Proof. Differentiating relation (14), from the proof of Theorem 1, we have

$$
\beta M^{\prime}(z)+z M^{\prime \prime}(z)=\beta \phi^{\gamma-1}(u, v ; z)\left[\phi(u, v ; z)+\gamma z \phi^{\prime}(u, v ; z)\right]
$$

which is equivalent to

$$
M^{\prime}(z)\left[(\beta-1)+1+\frac{z M^{\prime \prime}(z)}{M^{\prime}(z)}\right]=\beta \phi^{\gamma-1}(u, v ; z)\left[\phi(u, v ; z)+\gamma z \phi^{\prime}(u, v ; z)\right]
$$

Let

$$
1+\frac{z M^{\prime \prime}(z)}{M^{\prime}(z)}=p(z), z \in U, p(0)=1
$$

By replacing (32) in (31) we obtain

$$
M^{\prime}(z)[(\beta-1)+p(z)]=\beta \phi^{\gamma-1}(u, v ; z)\left[\phi(u, v ; z)+\gamma z \phi^{\prime}(u, v ; z)\right]
$$

Differentiating relation (33) we get

$$
\frac{z M^{\prime \prime}(z)}{M^{\prime}(z)}+\frac{z p^{\prime}(z)}{\beta-1+p(z)}=(\gamma-1) \frac{z \phi^{\prime}(u, v ; z)}{\phi(u, v ; z)}+\frac{z(\gamma+1) \phi^{\prime}(u, v ; z)+z^{2} \gamma \phi^{\prime \prime}(u, v ; z)}{\phi(u, v ; z)+\gamma z \phi^{\prime}(u, v ; z)} \text {. }
$$

After some computations, we have

$$
1+\frac{z M^{\prime \prime}(z)}{M^{\prime}(z)}+\frac{z p^{\prime}(z)}{\beta-1+p(z)}=1+(\gamma-1) \frac{z \phi^{\prime}(u, v ; z)}{\phi(u, v ; z)}+\frac{z(\gamma+1) \phi^{\prime}(u, v ; z)+z^{2} \gamma \phi^{\prime \prime}(u, v ; z)}{\phi(u, v ; z)+\gamma z \phi^{\prime}(u, v ; z)}
$$

Using relation (32) in (34) we can write

$$
p(z)+\frac{z p^{\prime}(z)}{\beta-1+p(z)}=E(u, v ; z), z \in U .
$$


By considering (35) in (30), the following inequality emerges

$$
\frac{\left|p(z)+\frac{z p^{\prime}(z)}{\beta-1+p(z)}\right|}{1+\left|p(z)+\frac{z p^{\prime}(z)}{\beta-1+p(z)}\right|} \leq \frac{\left|(\beta-1)\left(1-z^{2}\right)+\gamma(1+z)^{2}\right|}{\left|(\beta-1)(1-z)^{2}+\gamma\left(1-z^{2}\right)\right|+\left|(\beta-1)\left(1-z^{2}\right)+\gamma(1+z)^{2}\right|} .
$$

In order to obtain the expected result, Lemma 1 will be used. For that, let $\psi$ : $\mathbb{C}^{2} \times U \rightarrow \mathbb{C}$, given by (21) and for $r=p(z)$ and $s=z p^{\prime}(z)$ from relation (35), we have

$$
\psi\left(p(z), z p^{\prime}(z)\right)=E(u, v ; z), \quad z \in U .
$$

Using (37) in (30), we get

$$
\frac{\left|\psi\left(p(z), z p^{\prime}(z)\right)\right|}{1+\left|\psi\left(p(z), z p^{\prime}(z)\right)\right|} \leq \frac{\left|(\beta-1)\left(1-z^{2}\right)+\gamma(1+z)^{2}\right|}{\left|(\beta-1)(1-z)^{2}+\gamma\left(1-z^{2}\right)\right|+\left|(\beta-1)\left(1-z^{2}\right)+\gamma(1+z)^{2}\right|} .
$$

Using Lemma 1 , for $\delta=\beta-1, \omega=\gamma \neq 0$, we have

$$
\psi\left(p(z), z p^{\prime}(z)\right) \prec_{\mathcal{F}} h(z)=\frac{(\beta-1)\left(1-z^{2}\right)+\gamma(1+z)^{2}}{(\beta-1)(1-z)^{2}+\gamma\left(1-z^{2}\right)}
$$

which, according to Lemma 1, implies

$$
p(z) \prec_{\mathcal{F}} q(z)=\frac{1+z}{1-z}, z \in U .
$$

Using in (40) relation (32) we have

$$
1+\frac{z M^{\prime \prime}(z)}{M^{\prime}(z)} \prec \mathcal{F} q(z)=\frac{1+z}{1-z}, z \in U .
$$

Since $q$ is convex, relation (41) is equivalent to

$$
\operatorname{Re}\left(1+\frac{z M^{\prime \prime}(z)}{M^{\prime}(z)}\right)>\operatorname{Re} \frac{1+z}{1-z}>0, z \in U \text {, hence } M \in \mathcal{K} .
$$

Remark 6. Using Lemma 3 and the convexity property proved for the operator $M(z)$, the following corollary can be stated giving the property of the integral operator $M(z)$ given by (7) to be starlike of order $\frac{1}{2}$.

Corollary 3. Let the operator $M(z)$ be given by (7). Then $M(z) \in \mathcal{K}$ implies $M(z) \in \mathcal{S}^{*}\left(\frac{1}{2}\right)$.

Proof. Since $\operatorname{Re}\left[1+\frac{z M^{\prime \prime}(z)}{M^{\prime}(z)}\right]>0$ using Lemma 3 we obtain $\operatorname{Re} \frac{z M^{\prime}(z)}{M(z)}>\frac{1}{2}$, hence $M \in$ $\mathcal{S}^{*}\left(\frac{1}{2}\right)$.

Remark 7. Using function $q(z)=\frac{1-2 z}{1+z}$ as fuzzy best dominant in Theorem 1, we get the following corollary, which gives a sufficient condition for the operator $M(z)$ given by (7) to be convex of order $\left(-\frac{1}{2}\right)$. 
Corollary 4. For $\beta, \gamma \in \mathbb{C}, \beta>1, \gamma>0$, let the fuzzy function $F: \mathbb{C} \rightarrow[0,1]$ given by (9) and consider the holomorphic function in $U$ given by the equation

$$
h(z)=\frac{(\beta-1)(1+z)(1-2 z)+(1-2 z)^{2}-3 z}{(\beta-1)(1+z)^{2}+(1-2 z)(1+z)},
$$

$h(0)=q(0)=1$, where function $q(z)=\frac{1-2 z}{1+z}$ is a univalent solution in $U$ which satisfies the fuzzy differential subordination

$$
\begin{gathered}
\frac{|1-2 z|}{|1+z|+|1-2 z|} \leq \\
\frac{\left|(\beta-1)(1+z)(1-2 z)+(1-2 z)^{2}-3 z\right|}{\left|(\beta-1)(1+z)^{2}+(1-2 z)(1+z)\right|+\left|(\beta-1)(1+z)(1-2 z)+(1-2 z)^{2}-3 z\right|},
\end{gathered}
$$

i.e.,

$$
\frac{1-2 z}{1+z} \prec_{\mathcal{F}} \frac{(\beta-1)(1+z)(1-2 z)+(1-2 z)^{2}-3 z}{(\beta-1)(1+z)^{2}+(1-2 z)(1+z)}, z \in U .
$$

Consider the confluent (or Kummer) hypergeometric function $\phi(u, v ; z)$ given by (5), and the operator $M(z)$ given by (7).

If

$$
E(u, v ; z)=1+(\gamma-1) \frac{z \phi^{\prime}(u, v ; z)}{\phi(u, v ; z)}+\frac{(\gamma+1) z \phi^{\prime}(u, v ; z)+z^{2} \gamma \phi^{\prime \prime}(u, v ; z)}{\phi(u, v ; z)+\gamma z \phi^{\prime}(u, v ; z)}
$$

is analytic in $U$, and

$$
\begin{gathered}
\frac{|E(u, v ; z)|}{1+|E(u, v ; z)|} \leq \\
\frac{\left|(\beta-1)(1+z)(1-2 z)+(1-2 z)^{2}-3 z\right|}{\left|(\beta-1)(1+z)^{2}+(1-2 z)(1+z)\right|+\left|(\beta-1)(1+z)(1-2 z)+(1-2 z)^{2}-3 z\right|} \\
\text { i.e., } E(u, v ; z) \prec_{\mathcal{F}} \frac{(\beta-1)(1+z)(1-2 z)+(1-2 z)^{2}-3 z}{(\beta-1)(1+z)^{2}+(1-2 z)(1+z)},
\end{gathered}
$$

then

$$
\frac{\left|1+\frac{z M^{\prime \prime}(z)}{M^{\prime}(z)}\right|}{1+\left|1+\frac{z M^{\prime \prime}(z)}{M^{\prime}(z)}\right|} \leq \frac{\left|\frac{1-2 z}{1+z}\right|}{1+\left|\frac{1-2 z}{1+z}\right|} \text { i.e., } 1+\frac{z M^{\prime \prime}(z)}{M^{\prime}(z)} \prec_{\mathcal{F}} \frac{1-2 z}{1+z}, z \in U \text {, or } M \in \mathcal{K}\left(-\frac{1}{2}\right) \text {, }
$$

and $q(z)=\frac{1-2 z}{1+z}$ is the fuzzy best dominant.

Proof. Using in (41) $q(z)=\frac{1-2 z}{1+z}$, the relation becomes

$$
1+\frac{z M^{\prime \prime}(z)}{M^{\prime}(z)} \prec_{\mathcal{F}} \frac{1-2 z}{1+z}, z \in U
$$

Since $\operatorname{Re}\left(\frac{z q^{\prime \prime}(z)}{q^{\prime}(z)}+1\right)=\operatorname{Re} \frac{1-z}{1+z}>0$, we have that $q$ is convex and $\operatorname{Re} \frac{1-2 z}{1+z}=-\frac{1}{2}$. Then relation (43) is equivalent to

$$
\operatorname{Re}\left(1+\frac{z M^{\prime \prime}(z)}{M^{\prime}(z)}\right)>\operatorname{Re} \frac{1-2 z}{1+z}>-\frac{1}{2}, z \in U, \text { hence } M \in \mathcal{K}\left(-\frac{1}{2}\right) .
$$


Remark 8. Using Corollary 4 we next prove that the operator $M(z)$ given by $(7)$ is close-to-convex in $U$.

Theorem 2. Let $M(z)$ be given by (7) satisfying the condition $\operatorname{Re}\left(1+\frac{z M^{\prime \prime}(z)}{M^{\prime}(z)}\right)>-\frac{1}{2}, z \in U$. Then $M(z)$ is close-to-convex.

Proof. For obtaining the desired result, Lemma 2 is applied. We calculate

$$
\int_{\theta_{1}}^{\theta_{2}} \operatorname{Re}\left(1+\frac{z M^{\prime \prime}(z)}{M^{\prime}(z)}\right) d \theta=\int_{\theta_{1}}^{\theta_{2}}-\frac{1}{2} d \theta=-\left.\frac{1}{2} \theta\right|_{\theta_{1}} ^{\theta_{2}}=-\frac{1}{2}\left(\theta_{2}-\theta_{1}\right)>-\frac{1}{2} 2 \pi=-\pi .
$$

From Lemma 2, this means that $M(z) \in \mathcal{C}$.

Example 1. Let $u=-1, v=\frac{1}{3}, \phi\left(-1, \frac{1}{3} ; z\right)=1+3 i z$, and

$$
\frac{z \phi^{\prime}\left(-1, \frac{1}{3} ; z\right)}{\phi\left(-1, \frac{1}{3} ; z\right)}=\frac{3 i z}{1+3 i z}=1-\frac{1-3 \sin \alpha-3 i \cos \alpha}{10-6 \sin \alpha} \text {. }
$$

For $\beta=2, \gamma=1$, we calculate

$$
M(z)=\frac{2}{z} \int_{0}^{z}(1+3 i t) t d t=\frac{2}{z}\left(\frac{z^{2}}{2}+i z^{3}\right)=z+2 i z^{2},
$$

and $\frac{z M^{\prime}(z)}{M(z)}=1+\frac{2 i z}{1+2 i z}$.

Using Corollary 1, we have:

Let $\beta=2, \gamma=1$, fuzzy function $F(z)=\frac{|z|}{1+|z|}, z \in U$, and $h(z)=\frac{1-2 z}{1+z}, h(0)=1$, with univalent solution $q(z)=\frac{1-z}{1+z}, z \in U$, which satisfy the fuzzy differential subordination

$$
\frac{|1-z|}{|1+z|+|1-z|} \leq \frac{|1-2 z|}{|1+z|+|1-2 z|}, \text { i.e., } q(z)=\frac{1-z}{1+z} \prec \mathcal{F} \frac{1-2 z}{1+z}=h(z),
$$

and $\phi\left(-1, \frac{1}{3} ; z\right)=1+3 i z$, Kummer hypergeometric function.

If $\frac{1+6 i z}{1+3 i z}$ is holomorphic in $U$, and

$$
\frac{|1+6 i z|}{|1+3 i z|+|1+6 i z|} \leq \frac{|1-2 z|}{|1+z|+|1-2 z|}, \text { i.e., } \frac{1+6 i z}{1+3 i z} \prec \mathcal{F} \frac{1-2 z}{1+z},
$$

then

$$
\frac{|3 i z|}{|1+3 i z|+|3 i z|} \leq \frac{|1-z|}{|1+z|+|1-z|} \text {, i.e., } \frac{3 i z}{1+3 i z} \prec \mathcal{F} \frac{1-z}{1+z}, z \in U \text {. }
$$

In

$$
\begin{aligned}
& \operatorname{Re} \frac{z \phi^{\prime}\left(-1, \frac{1}{3} ; z\right)}{\phi\left(-1, \frac{1}{3} ; z\right)}=\operatorname{Re}\left(1-\frac{1-3 \sin \alpha-3 i \cos \alpha}{10-6 \sin \alpha}\right)=1-\frac{1-3 \sin \alpha}{10-6 \sin \alpha} \\
= & \frac{10-6 \sin \alpha-1+3 \sin \alpha}{4+6(1-\sin \alpha)}=\frac{9-3 \sin \alpha}{4+6(1-\sin \alpha)}=\frac{6+3(1-\sin \alpha)}{4+6(1-\sin \alpha)}>0 .
\end{aligned}
$$

\section{Conclusions}

Since the study of operators using the fuzzy differential subordination theory presents interest at this time, and many new and interesting results have been obtained recently, the research regarding this topic is further conducted in this paper. A new hypergeometric integral operator $M(z)$ is introduced in this paper in relation (7) by using a confluent (or Kummer) hypergeometric function and, having as inspiration, the operator studied by Miller, Mocanu and Reade in 1978 [28] and taking specific values for parameters $\beta, \gamma, \alpha, \delta$ 
involved in its definition. Using the notion of fuzzy differential subordination and results related to it, in the first theorem proved, the fuzzy best dominant of a certain fuzzy differential subordination is given. Using particular functions as fuzzy best dominants, several corollaries are stated, giving sufficient conditions for the operator $M(z)$ to be starlike, convex, starlike of order $\frac{1}{2}$ and convex of order $\left(-\frac{1}{2}\right)$, respectively. The second theorem proved shows the property of the operator $M(z)$ to be close-to-convex. For further study, the properties already proved, related to starlikeness and convexity of the operator $M(z)$, could inspire applications in introducing special classes of analytic functions. The operator could also be studied using the dual theory of fuzzy differential superordination, possibly obtaining sandwhich-type theorems, connecting with the present results a usual outcome in geometric function theory. Since particular values for parameters have been used for defining this operator, it might be interesting to try using other values for obtaining certain potentially interesting operators. It being well-known how hypergeometric functions have numerous applications in physics, engineering and statistics, applications of the operators involving those functions could prove useful in other disciplines. The theory of fuzzy differential subordination is still very new and one cannot predict what applications in real life or other scientific domains it might have. Those are subjects for investigation in long-term future studies.

Funding: This research received no external funding.

Institutional Review Board Statement: Not applicable.

Informed Consent Statement: Not applicable.

Data Availability Statement: Not applicable.

Conflicts of Interest: The author declares no conflict of interest.

\section{References}

1. Zadeh, L.A. Fuzzy Sets. Inf. Control 1965, 8, 338-353. [CrossRef]

2. Dzitac, S.; Nădăban, S. Soft Computing for Decision-Making in Fuzzy Environments: A Tribute to Professor Ioan Dzitac. Mathematics 2021, 9, 1701. [CrossRef]

3. Dzitac, I. Zadeh's Centenary. Int. J. Comput. Commun. Control 2021, 16, 4102. [CrossRef]

4. Oros, G.I.; Oros, G. The notion of subordination in fuzzy sets theory. Gen. Math. 2011, 19, 97-103.

5. Miller, S.S.; Mocanu, P.T. Second order-differential inequalities in the complex plane. J. Math. Anal. Appl. 1978, 65, $298-305$. [CrossRef]

6. Miller, S.S.; Mocanu, P.T. Differential subordinations and univalent functions. Michig. Math. J. 1981, 28, 157-171. [CrossRef]

7. Oros, G.I.; Oros, G. Fuzzy differential subordination. Acta Univ. Apulensis 2012, 3, 55-64.

8. Oros, G.I.; Oros, G. Dominants and best dominants in fuzzy differential subordinations. Stud. Univ. Babeş-Bolyai Math. 2012, 57, 239-248.

9. Oros, G.I.; Oros, G. Briot-Bouquet fuzzy differential subordination. An. Univ. Oradea Fasc. Mat. 2012, $19,83-87$.

10. Dzitac, I.; Filip, F.G.; Manolescu, M.J. Fuzzy Logic Is Not Fuzzy: World-renowned Computer Scientist Lotfi A. Zadeh. Int. J. Comput. Commun. Control 2017, 12, 748-789. [CrossRef]

11. Atshan, W.G.; Hussain, K.O. Fuzzy Differential Superordination. Theory Appl. Math. Comput. Sci. 2017, 7, 27-38.

12. Alb Lupaş, A. A note on special fuzzy differential subordinations using generalized Sălăgean operator and Ruscheweyh derivative. J. Comput. Anal. Appl. 2013, 15, 1476-1483.

13. Alb Lupaş, A.; Oros, G. On special fuzzy differential subordinations using Sălăgean and Ruscheweyh operators. Appl. Math. Comput. 2015, 261, 119-127.

14. Venter, A.O. On special fuzzy differential subordination using Ruscheweyh operator. Analele Univ. Oradea Fasc. Mat. 2015, 22, 167-176.

15. Eş, A.H. On fuzzy differential subordination. Math. Moravica 2015, 19, 123-129.

16. Majeed, A.H. Fuzzy differential subordinations properties of analytic functions involving generalized differential operator. Sci. Int. Lahore 2018, 30, 297-302.

17. Ibrahim, R.W. On the subordination and superordination concepts with applications. J. Comput. Theor. Nanosci. 2017, 14, 2248-2254. [CrossRef]

18. Altai, N.H.; Abdulkadhim, M.M.; Imran, Q.H. On first order fuzzy differential superordination. J. Sci. Arts 2017, 173, 407-412.

19. Thilagavathi, K. Fuzzy subordination and superordination results for certain subclasses of analytic functions associated with Srivastava-Attitya operator. Int. J. Pure Appl. Math. 2018, 118, 921-929. 
20. El-Deeb, S.M.; Alb Lupaş, A. Fuzzy differential subordinations associated with an integral operator. An. Univ. Oradea Fasc. Mat. 2020, 27, 133-140.

21. Alb Lupaş, A.; Oros, G.I. New Applications of Sălăgean and Ruscheweyh Operators for Obtaining Fuzzy Differential Subordinations. Mathematics 2021, 9, 2000. [CrossRef]

22. El-Deeb, S.M.; Oros, G.I. Fuzzy differential subordinations connected with the linear operator. Math. Bohem. 2021, 1-10. [CrossRef]

23. Oros, G.I. New fuzzy differential subordinations. Commun. Fac. Sci. Univ. Ank. Ser. A1 Math. Stat. 2021, 70, $229-240$.

24. Srivastava, H.M.; El-Deeb, S.M. Fuzzy Differential Subordinations Based upon the Mittag-Leffler Type Borel Distribution. Symmetry 2021, 13, 1023. [CrossRef]

25. Miller, S.S.; Mocanu, P.T. Differential Subordinations. Theory and Applications; Marcel Dekker, Inc.: New York, NY, USA; Basel, Switzerland, 2000.

26. de Branges, L. A proof of the Bieberbach conjecture. Acta Math. 1985, 154, 137-152. [CrossRef]

27. Srivastava, H.M. Operators of basic (or q-) calculus and fractional q-calculus and their applications in geometric function theory of complex analysis. Iran. J. Sci. Technol. Trans. A Sci. 2020, 44, 327-344. [CrossRef]

28. Miller, S.S.; Mocanu, P.T.; Reade, M.O. Starlike integral operators. Pac. J. Math. 1978, 79, 157-168. [CrossRef]

29. Al-Janaby, H.F.; Ghanim, F. A subclass of Noor-type harmonic p-valent functions based on hypergeometric functions. Kragujev. J. Math. 2021, 45, 499-519. [CrossRef]

30. Alb Lupaş, A.; Oros, G.I. Differential Subordination and Superordination Results Using Fractional Integral of Confluent Hypergeometric Function. Symmetry 2021, 13, 327. [CrossRef]

31. Ghanim, F.; Al-Shaqsi, K.; Darus, M.; Al-Janaby, H.F. Subordination Properties of Meromorphic Kummer Function Correlated with Hurwitz-Lerch Zeta-Function. Mathematics 2021, 9, 192. [CrossRef]

32. Oros, G.I. Study on new integral operators defined using confluent hypergeometric function. Adv. Differ. Equ. 2021, $2021,342$. [CrossRef]

33. Mocanu, P.T.; Bulboacă, T.; Sălăgean, Ş.G. Geometric Theory of Analytic Functions; Casa Cărţii de Ştiinâă: Cluj-Napoca, Romania, 1999. 